4. E. Kasner, Conformal geometry, Proc. Fifth Internat. Congress Math., vol. 2,1912, p. 83 .

5. G. Königs, Ann. Ecole. Norm 3 (1884), no. 1.

DEPARTMENT OF MATHEMATICS, UNIVERSITY OF WISCONSIN, MADISON, WISCONSIN 53706

Current address: Department of Mathematics, University of Alabama in Huntsville, Huntsville, Alabama 35807

PROCEEDINGS OF THE

AMERICAN MATHEM ATICAL SOCIETY

Volume 50, July 1975

\title{
ON QUASINORMAL TOEPLITZ OPERATORS
}

\section{ICHIRO AMEMIYA, TAKASHI ITO ${ }^{1}$ AND TIN KIN WONG}

ABSTRACT. It is proved in this note that a quasinormal Toeplitz operator is either normal or analytic.

1. Introduction and the Theorem. Let $L^{p}$ be the Lebesgue space on the unit circle and let $H^{p}$ be the corresponding Hardy space for $1 \leq p \leq+\infty$. The Toeplitz operator $T_{\phi}$ with symbol $\phi$ in $L^{\infty}$ is the operator on $H^{2}$ defined by $T_{\phi} f=P(\phi f)$ for $f \in H^{2}$, where $P$ is the orthogonal projection of $L^{2}$ onto $H^{2}$. A Toeplitz operator $T_{\phi}$ is analytic if its symbol $\phi$ is in $H^{\infty}$. In this case $T_{\phi}$ is simply the multiplication on $H^{2}$ by $\phi$ with the multiplication on $L^{2}$ by $\phi$ as its normal extension. Thus any analytic Toeplitz operator is subnormal. We recall that an operator is subnormal if it is the restriction of some normal operator to one of its invariant subspaces. P. R. Halmos [2] raised the following interesting question: Is every subnormal Toeplitz operator either normal or analytic? The answer is not known yet. Some partial results are given in [3]. As a test question for this problem one may ask if quasinormal Toeplitz operators are either normal or analy-

Received by the editors January 21, 1974 and, in revised form, March 27, 1974. AMS (MOS) subject classifications (1970). Primary 47B20, 47B35.

Key words and phrases. Toeplitz operator, quasinormal operator, subnormal operator.

${ }^{1}$ Research of the second author was partially supported by the National Science Foundation under Grant No. GP-20150. 
tic. We recall that an operator $T$ is quasinormal if $T$ commutes with $T^{*} T$, and also recall that every quasinormal operator is subnormal. The purpose of this note is to answer this test question.

Theorem. A quasinormal Toeplitz operator is either normal or analytic.

2. Proof of the Theorem. Before the proof we need to introduce several notations and need to show some identities which are fundamental for our proof.

The functions $e_{n}(z)=z^{n}, n=0, \pm 1, \pm 2, \cdots$, form the usual orthonormal basis for $L^{2}$. The $n$th Fourier coefficient of $f$ is denoted by $\hat{f}(n)$; $f=\Sigma_{n=-\infty}^{+\infty} \hat{f}(n) e_{n}$ in $L^{2}$. For elements $g$ and $b$ of $H^{2}, g \otimes b$ denotes the rank-one operator on $H^{2} ; f \rightarrow\langle f, g\rangle, b$ where $\langle f, g\rangle$ is the inner product on $H^{2}$. The unilateral shift on $H^{2}$, namely, the multiplication by $e_{1}$, is denoted by $U$ instead of $T_{e_{i}}$.

For $\phi \in L^{\infty}$, define

$$
\phi_{+}=U^{*} T_{\phi} e_{0}=\sum_{n=0}^{\infty} \hat{\phi}(n+1) e_{n} \text { and } \phi_{-}=U^{*} T_{\phi}^{*} e_{0}=\sum_{n=0}^{\infty} \overline{\hat{\phi}(-n-1)} e_{n} .
$$

A simple computation (for example, by operating on the basis $e_{n}$ ) shows the following identities:

$$
T_{\phi} U-U T_{\phi}=\phi_{-} \otimes e_{0}, \quad T_{\phi}^{*} U-U T_{\phi}^{*}=\phi_{+} \otimes e_{0} .
$$

Using (1) and its adjoint we have

(2) $U^{*} T_{\phi}^{*} T_{\phi} U-T_{\phi}^{*} T_{\phi}=\phi_{-} \otimes \phi_{-}, \quad U^{*} T_{\phi} T_{\phi}^{*} U-T_{\phi} T_{\phi}^{*}=\phi_{+} \otimes \phi_{+}$.

Let $C_{\phi}$ be the self-commutator of $T_{\phi} ; C_{\phi}=T_{\phi}^{*} T_{\phi}-T_{\phi} T_{\phi}^{*}$, it follows immediately from (2) that

$$
U^{*} C_{\phi} U-C_{\phi}=\phi_{-} \otimes \phi_{-}-\phi_{+} \otimes \phi_{+} \cdot
$$

This identity shows that $\phi_{-} \otimes \phi_{-}=\phi_{+} \otimes \phi_{+}$is necessary and sufficient for $T_{\phi}$ to be normal. Because of (3), the necessity is trivial. Conversely, if $\phi_{-} \otimes \phi_{-}=\phi_{+} \otimes \phi_{+}$, then $\phi_{-}=\gamma \phi_{+}$for some number $\gamma$ with $|\gamma|=1$, that is $\overline{\phi(-n)}=\gamma \hat{\phi}(n)$ for all $n \neq 0$. Therefore $\phi$ becomes a linear function of a real valued function. Hence $T_{\phi}$ clearly commutes with $T_{\phi}^{*}=T_{\phi}$.

We add one more identity which we shall need later and which can be shown by combining (1) and (3).

(4) $U^{*} C_{\phi} T_{\phi} U-C_{\phi} T_{\phi}=\phi_{-} \otimes U^{*} C_{\phi} e_{0}+\left(\phi_{-} \otimes \phi_{-}-\phi_{+} \otimes \phi_{+}\right) T_{\phi}$. 
We can now start our proof.

Proof of the Theorem. Let $T_{\phi}$ be quasinormal but let it be neither normal nor analytic. Under these assumptions, the first half of our argument will be showing that $\phi_{-}$is an eigenvector of $T_{\phi}^{*} T_{\phi}$. Then using this fact, the rest of the argument will lead to a contradiction.

Since $T_{\phi}$ is quasinormal, that is $C_{\phi} T_{\phi}=0$, the identity (4) becomes

$$
\phi_{-} \otimes U^{*} C_{\phi} e_{0}=\left(\phi_{+} \otimes \phi_{+}-\phi_{-} \otimes \phi_{-}\right) T_{\phi} .
$$

The operator $S=\phi_{+} \otimes \phi_{+}-\phi_{-} \otimes \phi_{-}$is a nonzero operator, because $T_{\phi}$ is not normal. Hence the range of $S$ is the subspace spanned by $\phi_{+}$and $\phi_{-}$. The adjoint of (5): $U^{*} C_{\phi} e_{0} \otimes \phi_{-}=T_{\phi}^{*} S$ shows that $T_{\phi}^{*}$ maps $\phi_{+}$ and $\phi_{-}$into the one-dimensional subspace spanned by $\phi_{-}$. Thus there are numbers $\alpha$ and $\beta$ such that

$$
T_{\phi}^{*} \phi_{+}=\alpha \phi_{-} \quad \text { and } \quad T_{\phi}^{*} \phi_{-}=\beta \phi_{-} .
$$

If we substitute (6) into the adjoint of (5), and note that $\phi_{-} \neq 0$ because $T_{\phi}$ is not analytic, then we come to

$$
U^{*} C_{\phi} e_{0}=\bar{\alpha} \phi_{+}-\bar{\beta} \phi_{-} \text {. }
$$

Using (1) and its adjoint, it follows that

$$
T_{\phi}^{*} \phi_{+}-T_{\phi} \phi_{-}=U^{*} C_{\phi} e_{0}+\overline{\hat{\phi}(0)} \phi_{+}-\hat{\phi}(0) \phi_{-} \text {. }
$$

Substituting (6) and (7) into this equation, we have

$$
T_{\phi} \phi_{-}=(\alpha+\bar{\beta}+\hat{\phi}(0)) \phi_{-}-(\bar{\alpha}+\overline{\hat{\phi}(0)}) \phi_{+} \cdot
$$

Operating this equation by $T_{\phi}^{*}$ and using (6) again, we conclude that

$$
T_{\phi}^{*} T_{\phi} \phi_{-}=\lambda \phi_{-} \text {, }
$$

where $\lambda=\alpha \beta-|\alpha|^{2}+|\beta|^{2}-\overline{\hat{\phi}(0)} \alpha+\hat{\phi} /(0) \beta$.

Now we can show that this will lead to a contradiction to the nonanalyticity of $T_{\phi}$ by arguing in the following way. Let $K$ be the kernel of the operator $T_{\phi}^{*} T_{\phi}-\lambda I$. First, note that $K$ is a nonzero and proper subspace of $H^{2}$. Because of $\phi_{-} \neq 0, K$ is nonzero by (8) and $K$ is proper by (2). $K$ reduces $T_{\phi}$ because $T_{\phi}$ is quasinormal. Lastly, $K$ is invariant under the backward shift $U^{*}$. To see this, we have

$$
\left(T_{\phi}^{*} T_{\phi}-\lambda I\right) U^{*}=U^{*}\left(T_{\phi}^{*} T_{\phi}-\lambda I\right)-\left(T_{\phi}^{*} e_{0}\right) \otimes \phi_{-}-e_{0} \otimes T_{\phi}^{*} \phi_{+}
$$


by (1) and its adjoint. This equation and the fact that $T_{\phi \phi_{+}}^{*}=\alpha \phi_{-}$with $\phi_{-} \in K$ imply $\left(T_{\phi}^{*} T_{\phi}-\lambda I\right)^{2} U^{*}(K)=\{0\}$. Hence $U^{*}(K) \subset K$.

The orthogonal complement $K^{\perp}$ of $K$ now becomes a nonzero and proper subspace which is invariant under both the shift $U$ and $T_{\phi^{*}}$. The well-known Beurling's characterization of invariant subspaces of the shift $U$ gives $K^{\perp}=\chi^{2}$ for some nonconstant inner function $\chi$. Since $T_{\phi}$ leaves $\chi H^{2}$ invariant, it is not hard to see that $T_{\chi} T_{\phi}=T_{\phi} T_{\chi}$. The commutativity of $T_{\boldsymbol{\chi}}$ and $T_{\phi}$ implies that $T_{\phi}$ must be analytic [1], contradicting the assumption which we started with.

3. Concluding remarks. We conclude this note with two remarks.

Remark 1. It is easy to see that a quasinormal analytic Toeplitz operator is of the form $T_{\alpha \chi}$ where $X$ is an inner function and $\alpha$ is a number [3]. Here is a direct proof. If $T_{\phi}$ is quasinormal with $\phi \in H^{\infty}$, then the equation $T_{\phi}^{*} T_{\phi} T_{\phi} e_{0}=T_{\phi} T_{\phi}^{*} T_{\phi} e_{0}$ implies that $\left\|T_{\phi} \phi\right\|=\left\|T_{\phi}^{*} \phi\right\|$ which in turn implies that $\left\||\phi|^{2}\right\|=\left\|P\left(|\phi|^{2}\right)\right\|$. So the real valued function $|\phi|^{2}$ is in $H^{2}$. Therefore $|\phi|^{2}=\alpha^{2}$ for some constant $\alpha$. Consequently $\phi=\alpha \chi$ with $\chi=\phi /|\phi|$ inner. The argument in the paragraph right after identity (3) shows that any normal Toeplitz operator must have a linear function of some real valued function as its symbol. This is of course the characterization of normal Toeplitz operators by Brown and Halmos [1]. The theorem can now be rephrased as follows:

The class of quasinormal Toeplitz operators is exactly the class of Toe. plitz operators whose symbols are either linear functions of real valued func. tions or constant multiples of inner functions.

Remark 2. We consider the class of subnormal Toeplitz operators whose self-commutators are of rank one. In general, if $T$ is an operator such that $T^{*}$ acts as a scalar on the range of its self-commutator, that is

$$
T^{*}\left(T^{*} T-T T^{*}\right)=\lambda\left(T^{*} T-T T^{*}\right)
$$

for a number $\lambda$, then $T-\bar{\lambda} I$ is quasinormal. Note that $T-\bar{\lambda} I$ and $T$ have the same self-commutator. In particular, if $T$ is a subnormal operator with rank-one self-commutator then $T-\lambda$ is quasinormal because $T^{*}$ leaves invariant the range of its self-commutator for such subnormal $T$. It is then an immediate consequence of the Theorem that we have the following fact:

If $T_{\phi}$ is a subnormal Toeplitz operator with rank-one self-commutator, then its symbol $\phi$ is a linear function of some inner function $\chi$, where $\chi(z)=(z-\alpha) /(1-\bar{\alpha} z)$ for some $|\alpha|<1$. 
We note the inner function $\chi$ is of the form because the self-commutator $C_{\phi}$ is of rank one.

We are informed by the editor that $\mathrm{K}$. Clancy has shown more generally that any pure subnormal operator with rank-one self-commutator is a linear function of the unilateral shift (see Indiana Univ. Math. J. 23 (1973), 497-511).

\section{REFERENCES}

1. A. Brown and P. R. Halmos, Algebraic properties of Toeplitz operators, J. Reine Angew. Math. 213 (1963/64), 89-102. MR 28 \#3350; erratum MR 30, 1205.

2. P. R. Halmos, Ten problems in Hilbert space, Bull. Amer. Math. Soc. 76 (1970), 887-933. MR 42 \# 5066.

3. Takashi Ito and Tin Kin Wong, Subnormality and quasinormality of Toeplitz operators, Proc. Amer. Math. Soc. 34 (1972), 157-164. MR 46 \# 2472.

DEP ARTMENT OF MATHEMATICS, TOKYO INSTITUTE OF TECHNOLOGY, TOKYO, J AP AN (Current address of Ichiro Amemiya)

DEPARTMENT OF MATHEMATICS, WAYNE STATE UNIVERSITY, DETROIT, MICHIGAN 48202 (Current address of Takashi Ito and Tin Kin Wong) 